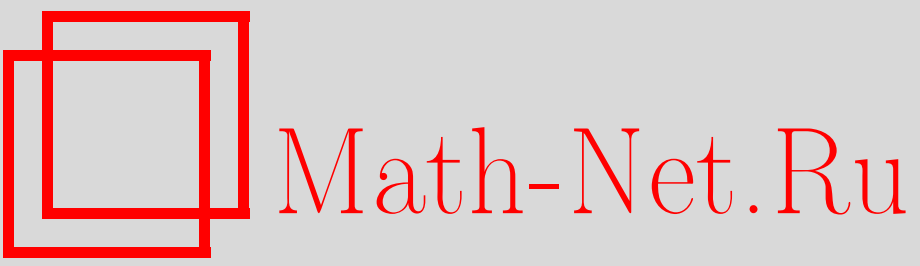

Э. Г. Оруджев, Спектральный анализ дифференциальных операторов с кратными характеристиками на полуоси, УМН, 1999, том 54, выпуск 2, 181-182

DOI: https://doi.org/10.4213/rm144

Использование Общероссийского математического портала Math-Net.Ru подразумевает, что вы прочитали и согласны с пользовательским соглашением

http://www.mathnet.ru/rus/agreement

Параметры загрузки:

IP: 54.198 .64 .247

26 апреля 2023 г., 10:52:19 


\title{
СПЕКТРАЛЬНЫЙ АНАЛИЗ ДИФФЕРЕНЦИАЛЬНЫХ ОПЕРАТОРОВ С КРАТНЫМИ ХАРАКТЕРИСТИКАМИ НА ПОЛУОСИ
}

\author{
Э.Г. ОрУдЖев
}

В данной работе исследуется спектр, резольвента, кратное разложение по собственным и присоединенным функциям дискретного спектра и по главным функциям непрерывного спектра дифференциального оператора

$$
l(Y) \equiv Y^{(2 n)}+\sum_{i=1}^{2 n} P_{i}(x, \lambda) Y^{(2 n-i)}, \quad P_{i}(x, \lambda)=\sum_{k=0}^{i} \lambda^{i-k} P_{i, i-k}(x), \quad i=\overline{1,2 n},
$$

с нормированными краевыми условиями в нуле в пространстве $L_{2}(0, \infty)$.

Ранее подобные операторы изучались в [1], [2], в случае, когда дифференциальное выражение, определяющее оператор, при $\lambda \rightarrow \infty$ близко двучленному $Y^{(2 n)}-\lambda^{2 n} Y$. Случай кратных корней характеристического уравнения не был изучен. Ему и посвящена настоящая работа.

Рассмотрим на интервале $0 \leqslant x \leqslant \infty$ дифференциальное уравнение

$$
Y^{(2 n)}+P_{1}(x, \lambda) Y^{(2 n-1)}+\cdots+P_{2 n}(x, \lambda) Y=0
$$

где

$$
P_{i}(x, \lambda)=\sum_{k=0}^{i} P_{i k}(x) \lambda^{k}, \quad i=\overline{1,2 n}
$$

$P_{i i}, i=\overline{1,2 n},-$ постоянные числа, функции $P_{i k}(x)$ определены и непрерывны в $[0, \infty)$, интегрируемы в $[0, \infty)$ в предположении, что характеристическое уравнение

$$
\theta^{2 n}+P_{1,1} \theta^{2 n-1}+\cdots+P_{2 n-1,2 n-1} \theta+P_{2 n, 2 n}=0
$$

имеет два различных корня $\theta_{1}, \theta_{2}$, каждый кратности $n$, и $\operatorname{Re} \lambda \theta_{1} \leqslant 0 \leqslant \operatorname{Re} \lambda \theta_{2}$.

ТеОрема 1. Пусть выполняются следующие условия:

1) существуют производные $P_{i, i-1}^{(\nu)}(x), i=\overline{1,2 n}, \nu=\overline{0,2 n-1} ; P_{i, i-2}^{(\nu)}(x), i=\overline{2,2 n}$, $\nu=\overline{0,2 n-2}, \ldots, P_{i, i-n+1}^{(\nu)}(x), i=\overline{n-1,2 n}, \nu=\overline{0, n+1}$, непрерывные на множестве $a \leqslant x<\infty$, их предель при $x \rightarrow \infty$ конечны и

(4)

$$
\sum_{i=1}^{2 n} P_{i, i-1} \theta_{k}^{2 n-i}=0, \sum_{i=2}^{2 n} P_{i, i-2} \theta_{k}^{2 n-i}=0, \ldots, \sum_{i=n-1}^{2 n} P_{i, i-(n-1)} \theta_{k}^{2 n-i}=0, k=1,2
$$

2) для каждой области $|\lambda| \geqslant \Gamma, a \leqslant x<\infty$ интеграль

(5)

$$
\begin{gathered}
\frac{1}{\Gamma^{n-1}} \int_{a}^{\infty} \Gamma^{1+m}\left|P_{n, 0}^{(\nu)}(x)\right| d x, \quad m=0, \quad \nu=\overline{0, n}, \\
\frac{1}{\Gamma^{n}} \int_{a}^{\infty} \Gamma^{1+m}\left|P_{n+1, m}^{(\nu)}(x)\right| d x, \quad m=0,1, \quad \nu=\overline{0, n-1}, \\
\frac{1}{\Gamma^{n+1}} \int_{a}^{\infty} \Gamma^{1+m}\left|P_{n+2, m}^{(\nu)}(x)\right| d x, \quad m=0,1,2, \quad \nu=\overline{0, n-2}, \ldots, \\
\frac{1}{\Gamma^{2 n-1}} \int_{a}^{\infty} \Gamma^{1+m}\left|P_{2 n, m}(x)\right| d x, \quad m=\overline{0,1, \ldots, n},
\end{gathered}
$$

существуют и меньше единиць. 
Тогда дифференциальное уравнение (1) в каждой из полуплоскостей $\pi_{ \pm}=\left\{ \pm \theta_{k} \lambda\right.$ : $\left.\operatorname{Re} \theta_{k} \lambda \geqslant 0\right\}$ при всех $x \in[0, \infty)$ имеет асимптотические решения, представимые в виде:

(6) $\quad Y_{i}(x, \lambda)=\left[g_{i 0}^{(0)}(x)+\frac{1}{\lambda} g_{i 0}^{(1)}(x)+\cdots+\frac{1}{\lambda^{n-1}} g_{i 0}^{(n-1)}(x)+\frac{E(x, \lambda)}{\lambda^{n}}\right] e^{\theta_{k} \lambda x}$,

$k=1$ при $i=\overline{1, n} ; k=2$ при $i=\overline{n+1,2 n}$, где $g_{i 0}^{(0)}(x), i=\overline{1, n}, u g_{i 0}^{(0)}(x), i=\overline{n+1,2 n}$, образуют фундаментальную систему решений дифференциальных уравнений $n$-го порядка, $g_{i 0}^{(\nu)}(x), \nu=\overline{1, n-1}$, определены и непрерывны на интервале $[0, \infty)$ и имеют абсолютно непрерывные производнье до порядка $2 n-1$ включительно. Функция $E(x, \lambda)$ ограничена в области $a \leqslant x<\infty,|\lambda| \geqslant \Gamma$.

При $x \rightarrow \infty$ равномерно относительно $\lambda:\left\{\lambda \in \pi_{ \pm},|\lambda| \geqslant \Gamma\right\}$ уравнение (1) имеет ф.с.р., допускающую асимптотическое представление:

$$
Y_{i}(x, \lambda)=\left[g_{i 0}^{(0)}(x)+O(1)\right] e^{\theta_{k} \lambda x}, \quad x \rightarrow \infty, \quad \begin{aligned}
k & =1 \text { nрu } i=\overline{1, n}, \\
& k=2 \text { nрu } i=\overline{n+1,2 n} .
\end{aligned}
$$

Определим оператор $L_{\alpha}$ так: его область определения есть $D_{\alpha}$ и $L_{\alpha} Y=l(y)$ при $Y \in D$.

Рассмотрим полуплоскость $\pi_{-}\left(\operatorname{Re} \theta_{1} \lambda \leqslant 0\right)$. Обозначим $A_{n}(\lambda)=\operatorname{det}\left\|U_{i}\left(Y_{k}\right)\right\|_{i, k=1}^{n}$. При условии $\operatorname{Re} \theta_{1} \lambda<0$ все решения $Y_{k}(x, \lambda), k=\overline{1, n}$, принадлежат пространству $L_{2}(0, \infty)$, а на прямой $l_{1}=\left\{\lambda: \operatorname{Re} \lambda \theta_{1}=0\right\}$ ни одна $Y_{k}(x, \lambda), k=\overline{1, n}$, не принадлежит пространству $L_{2}(0, \infty)$. Как показано в [3], при предположении $A_{n}(\lambda) \neq 0$ каждая точка $\lambda$, лежащая на прямой $l_{1}$, принадлежит непрерывному спектру оператора $L_{\alpha}$, а дискретный спектр, состоящий из нулей определителя $A_{n}(\lambda)$, образует конечное множество. Если при $\lambda_{0} \in l_{1}$ имеет место $A_{n}\left(\lambda_{0}\right)=0$, то точка $\lambda_{0}$ является спектральной особенностью оператора $L_{\alpha}$.

Возьмем окружность $\Gamma_{N}=\{\lambda:|\lambda|=N\}$ большого радиуса $N$ с центром в начале координат. Положим $\Gamma_{N}=\Gamma_{N}^{\prime}+\Gamma_{N}^{\prime \prime}$, где $\Gamma_{N}^{\prime}-$ замкнутый контур в полуплоскости $\pi_{+}$, образованный полуокружностью $\Gamma_{N}$ и прямой $\operatorname{Re} \theta_{1} \lambda=+\varepsilon$, и $\Gamma_{N}^{\prime \prime}$ - контур, образованный полуокружностью, находящейся в полуплоскости $\pi_{-}$, и прямой $\operatorname{Re} \theta_{1} \lambda=-\varepsilon$.

Применяя методику из [2; c. 240-253], придем к теореме.

ТЕОрема 2. Пусть выполняются условия (4), (5), а собственные значения оператора $L_{\alpha}$ не попадают на непрерывный спектр. Пусть функции $\Phi_{i}(x), i=\overline{0,2 n-1}$, финитнь в окрестности нуля и бесконечности, дифференцируемы $(3 n-i)$ раза. Положим

$\Gamma(x, \lambda)=\lambda^{2 n-1} \Phi_{0}(x)+\cdots+\Phi_{2 n-1}(x)+\sum_{i=1}^{2 n} \sum_{l=1}^{2 n} P_{i l}(x) \frac{d^{2 n-i}}{d x^{2 n-i}}\left(\lambda^{l-1} \Phi_{0}(x)+\cdots+\Phi_{2 n-1}(x)\right)$.

Тогда имеет место формула 2 -кратного разложения

$$
\frac{\theta_{1}^{n} \theta_{2}^{n}}{2 \pi \sqrt{-1}} \lim _{\substack{N \rightarrow \infty \\ \varepsilon \rightarrow 0}} \int_{\Gamma_{N, \varepsilon}} \lambda^{s} d \lambda \int_{0}^{\infty} K(x, \xi, \lambda) \Gamma(\xi, \lambda) d \xi=\Phi_{s}(x), \quad s=\overline{0,2 n-1},
$$

сходимость равномерна при всех $x \in[0, \infty)$.

Автор благодарен академику АН Азербайджанской Республики М. Г. Гасымову за ценные советы.

\section{СПИСОК ЛИТЕРАТУРЫ}

[1] Фунтаков В.А. // Изв. АН Азерб. ССР. 1960. №6. С. 3-19; 1961. №1. С. 3-21. [2] Максудов Ф. Г. Пучки операторов с непрерывным спектром. Баку: ЭЛМ, 1992. [3] Оруджев Э. Г. // Труды ИММ АН Азербайджкана. 1997. Т. 6. С. 148-160.

Университет "Хазар", департамент "математика",

Принято редколлегией

г. Баку, Азербайджан

03.12 .1998

E-mail: hi@kuniv.Baku.az 